Proc. Indian Acad. Sci. (Earth Planet. Sci.) Vol. 98, No. 2, July 1989, pp. 207-211.

(C) Printed in India.

\title{
Phase space analysis of Indian summer monsoon rainfall over subdivisions
}

\author{
V SATYAN \\ Physical Research Laboratory, Ahmedabad 380009, India \\ MS received 21 December 1988; revised 15 April 1989
}

\begin{abstract}
Indian summer monsoon seasonal rainfall for the period 1871-1986 over the meteorological subdivisions has been analysed using the method of deterministic chaos. It is shown that a strange attractor underlies the monsoon evolution in each subdivision. The dimensionality of the attractor and the number of key variables necessary to model the subdivisional dynamics have been determined.
\end{abstract}

Keywords. Monsoon variability; deterministic chaos; strange attractors; subdivisional rainfall.

\section{Introduction}

In forced dissipative nonlinear systems long-term variability can arise from deterministic chaos or self-generated irregularity induced by nonlinear interactions in the system (Lorenz 1963, 1984). Such irregularity or aperiodicity in the system is associated with the existence of a strange attractor in the system phase space for a certain range of values of the forcing. The dimension of this attractor provides information on the intrinsic variability in the system and the dimension of the phase space in which it is embedded gives an upper bound to the number of dynamically relevant degrees of freedom. Recent progress in this area shows how one can reconstruct the multivariable long-term dynamics starting from a time series of a single variable of the system. In recent years this novel technique of analysing time series has been applied to some important meteorological systems. Nicholis and Nicholis (1984) studied the variability in the global paleoclimatic records using this method. Fraedrich (1986) estimated the dimensions of strange attractors associated with some midlatitude systems from the time series of surface pressure and sunshine duration. Hense (1987) investigated the possible existence of a strange attractor in the southern oscillation using the time series of Nauru rainfall, the sea surface temperatures at Puerto Chicama and the surface pressure at Darwin. Recently Satyan (1988) has examined whether a strange attractor underlies the evolution of Indian summer monsoon climate. He used the time series of all Indian summer monsoon seasonal rainfall derived from observations for the period 1871-1986. In all these studies it was found that a strange attractor describes the long-term dynamics of the system under consideration. In this paper we have analysed the time series of seasonal mean (June to September) rainfall in different meteorological subdivisions of India. Our purpose here is to study the dynamics of variability on the smaller spatial scale of subdivisions and compare it with the dynamics of large-scale variability. 


\section{Theory}

We have explained elsewhere (Satyan 1988) the basic concepts relating to attractors, their importance in modelling irregular phenomena, the significance of the dimensionality of attractors as well as its computation using the well-known algorithm due to Grassberger and Procaccia (1983). The essential idea here is to reconstruct the multivariable phase space for the system from the given time series of a single variable of the system and then determine the topological characteristics of the attractor associated with the dynamical system. Briefly, from the given time series one samples $n$ points separated by a fixed time delay $\tau$ so that one obtains points $X(t), X(t+\tau)$, $X(t+2 \tau), \cdots X(t+(n-1) \tau)$. By an appropriate choice of $\tau$, these points are expected to be linearly independent. An obvious choice is to choose $\tau$ greater than the autocorrelation decay time. We denote the $n$ points by a vector $\mathbf{X}(t)$. Let us next consider $M$ different instants of time $t_{i}, i=1, M$ and for each $t_{i}$ we obtain from the time series the vector $X_{i}=X\left(t_{i}\right) i=1, \ldots M$. From these $M$ vectors one calculates a correlation function of the attractor

$$
C(r)=\frac{1}{M^{2}} \sum_{\substack{i, j=1 \\ i \neq j}}^{M} \theta\left(r-\left|\mathbf{X}_{i}-\mathbf{X}_{j}\right|\right),
$$

where $\left|\mathbf{X}_{i}-\mathbf{X}_{j}\right|$ is the distance between the two points $i$ and $j$ in the $n$ dimensional vector space (the Euclidean norm) and $r$ is an arbitrary distance parameter. It should be noted that the points we have chosen on the attractor are random in time. However, they are spatially correlated as they all lie on the attractor. Thus $C(r)$ is a global measure of this spatial correlation. $\theta$ is the Heaviside function, $\theta(x)=1$ if $x>0, \theta(x)=0$ if $x<0$. Thus $C(r)$ simply counts the number of pairs of points whose distance $\left|\mathbf{X}_{i}-\mathbf{X}_{j}\right|$ is less than $r$. It has been shown (Grassberger and Procaccia 1983) that the dimensionality $d$ of the attractor is related to $C(r)$ by the relation

$$
C(r)=r^{d}(\text { for small } r)
$$

The basic idea here is that the number of points (and hence $C(r)$ of a $d$-dimensional attractor inside an $n$-dimensional ball of radius $r(d \leqslant n)$ scales as $r^{d}$. The slope of the curve $\log C(r)$ plotted against $\log (r)$ would then give the dimensionality $d$. In practice, one evaluates $d$ for different number $n$ of components for each vector $\mathbf{X}_{i}$. Then if the dynamics is governed by a low-dimensional attractor the slope $d$ saturates beyond a critical value $n_{c}$. This value of $d$ then gives the dimensionality of the attractor. The nearest integer above the saturation slope $d$ provides the minimum number of independent variables necessary to model the dynamics on the attractor. And $n_{c}$ provides an upper bound for the number of variables sufficient to model the dynamics. It should also be pointed out that if one analyses a series with "pure noise" then there would be no saturation in slope beyond reasonable values of $n_{c}$.

\section{Results and discussion}

We have analysed the time series of monsoon seasonal rainfall for the 29 meteorological subdivisions of India. Figure 1 shows these subdivisions. For each subdivision 


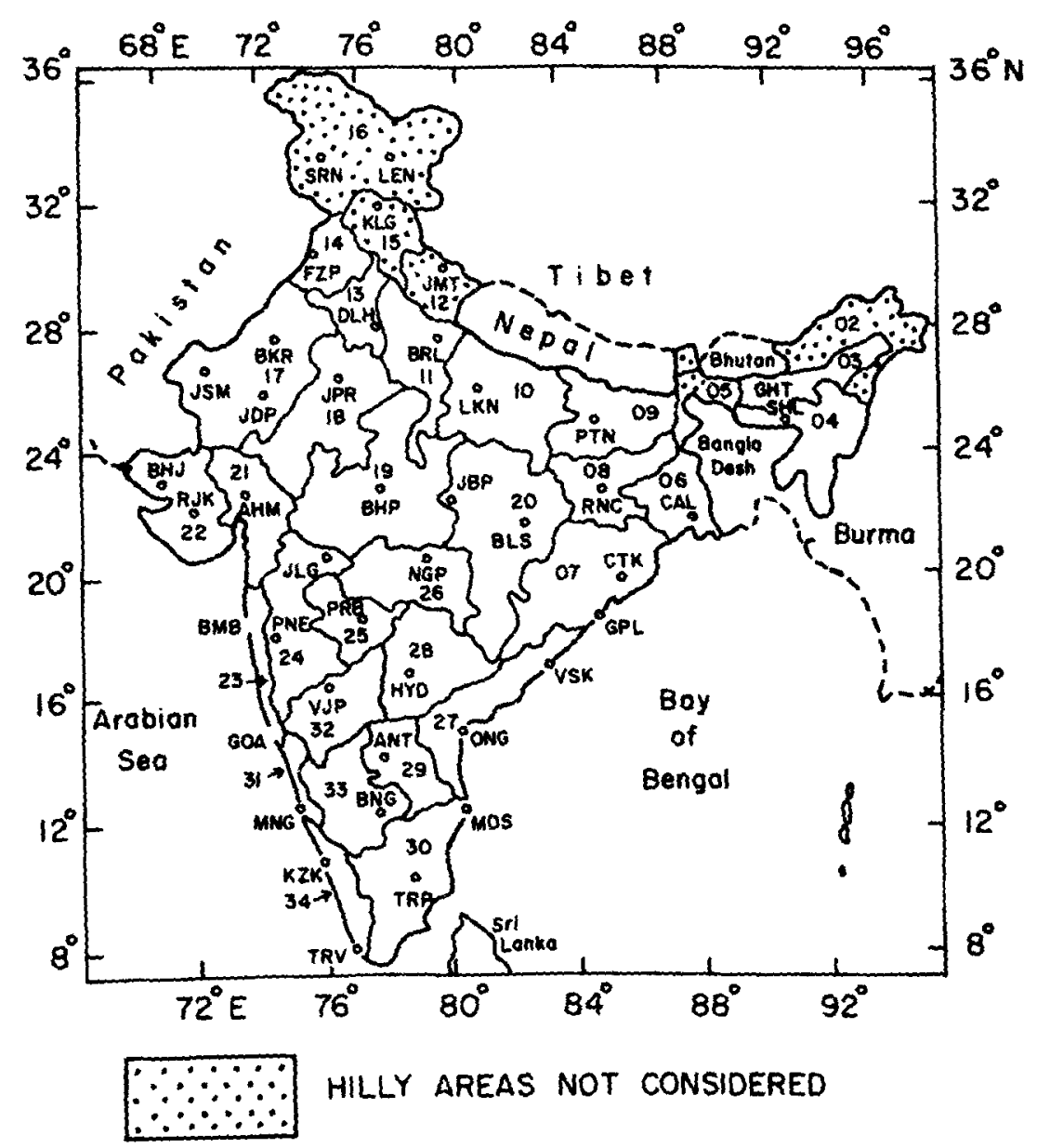

Figure 1. Metcorological subdivisions of contiguous India.

the time series used is the record of summer monsoon seasonal rainfall (June to September) for the period 1871 to 1986 derived from observations (Mooley and Parthasarathy 1984). For a range of values of the delay parameter $\tau$ (chosen so that it is larger than the decorrelation time of the time series) we carried out the following computations for each $\tau$. Beginning in a two-dimensional phase space (i.e. $n=2$ ) the correlation function for the time series was computed according to (1) for different values of the arbitrary distance parameter $r$. This procedure was repeated for increasing values of the embedding phase space dimension $(n=2$ to 10). The plots of $\ln (r)$ vs $\ln (\boldsymbol{r})$ for different subdivisions are not shown here. They are similar to the case of the all-India rainfall time series (viz figure 1 of Satyan 1988). Next for each curve with a particular value of $n$ we select a region on the curve, fit a straight line using a regression procedure and thus determine the slope in this region. This procedure is repeated for different regions on the curve. The scaling region then is the region which gives the best fit in the sense of having minimum residual variance. The slope corresponding to this fit gives the dimensionality of the attractor according to (2). The results of our analysis are given in table 1 which shows the different subdivisions and the dimensionality of attractor as determined in dimensions from 2 to 10 (slopes 
of $\ln C(r)$ vs $\ln (r)$ curves). We see that these slopes seem to converge as we go to higher embedding phase spaces and the mean of the saturation values of slope for the 29 subdivisions is seen to be 5.3. It is significant that this value is close to the saturation value of slope $5 \cdot 2$ for the all-India rainfall time series. This latter case is shown as subdivision $\# 0$ in table 1 . The fact that dimension is not an integer indicates the existence of a strange attractor governing the long-term dynamics. Essentially it implies that there is a large deterministic chaotic component in the observed variability on the spatial scale of subdivisions. The attractor dimensionalities for subdivisions range between the smallest value of 3.99 for the subdivision \#3, the eastern region, and the largest value of 6.30 for the subdivision \#21, the western region. In other words, the western region has a larger chaotic part in its variability compared to the eastern region. For the remaining subdivisions it is seen that their attractor dimensions are comparable to the dimension for the all-India rainfall series. Thus our results are

Table 1. Slope (attractor dimension) for different subdivisional rainfall time series in various embedding phase spaces. Subdivision 0 refers to the all-India rainfall series.

\begin{tabular}{|c|c|c|c|c|c|c|c|c|c|}
\hline \multirow[b]{2}{*}{ Subdivision } & \multicolumn{9}{|c|}{ Phase space dimension } \\
\hline & 2 & 3 & 4 & 5 & 6 & 7 & 8 & 9 & 10 \\
\hline 0 & 1.73 & $2 \cdot 43$ & 2.97 & $3 \cdot 17$ & $3 \cdot 64$ & $4 \cdot 13$ & $4 \cdot 73$ & $4 \cdot 84$ & $5 \cdot 17$ \\
\hline 3 & $1 \cdot 70$ & $2 \cdot 27$ & $2 \cdot 83$ & $3 \cdot 26$ & $3 \cdot 54$ & 3.55 & $3 \cdot 71$ & 3.96 & 3.99 \\
\hline 4 & 1.68 & $2 \cdot 39$ & 2.95 & $3 \cdot 32$ & 3.92 & $4 \cdot 42$ & $4 \cdot 45$ & 4.96 & $5 \cdot 50$ \\
\hline 5 & 1.76 & $2 \cdot 29$ & $2 \cdot 87$ & 3.45 & 3.94 & $4 \cdot 10$ & $4 \cdot 35$ & $4 \cdot 78$ & $5 \cdot 06$ \\
\hline 6 & $1 \cdot 76$ & $2 \cdot 46$ & 2.96 & $3 \cdot 45$ & $4 \cdot 07$ & $4 \cdot 37$ & $4 \cdot 75$ & $5 \cdot 12$ & $5 \cdot 62$ \\
\hline 7 & 1.71 & $2 \cdot 39$ & 3.03 & $3 \cdot 30$ & 3.89 & $4 \cdot 23$ & $4 \cdot 65$ & $4 \cdot 86$ & $5 \cdot 23$ \\
\hline 8 & 1.65 & $2 \cdot 13$ & 2.52 & $2 \cdot 91$ & $3 \cdot 42$ & $4 \cdot 01$ & $4 \cdot 56$ & 4.93 & $5 \cdot 27$ \\
\hline 9 & 1.75 & $2 \cdot 43$ & 2.90 & $3 \cdot 16$ & 3.73 & 3.99 & 4.45 & $5 \cdot 32$ & $5 \cdot 49$ \\
\hline 10 & 1.68 & $2 \cdot 36$ & $2 \cdot 91$ & 3.25 & $3-74$ & $4 \cdot 19$ & 4.43 & $4 \cdot 82$ & $5 \cdot 31$ \\
\hline 11 & 1.73 & 2.44 & 2.98 & 3.48 & 3.74 & $4 \cdot 18$ & 4.79 & $5 \cdot 21$ & 5.44 \\
\hline 13 & 1.81 & $2 \cdot 42$ & 3.02 & $3 \cdot 52$ & $3-89$ & $4 \cdot 10$ & $4 \cdot 68$ & $5 \cdot 30$ & $5 \cdot 88$ \\
\hline 14 & 1.82 & $2 \cdot 42$ & $2 \cdot 83$ & $3 \cdot 30$ & $3 \cdot 72$ & 3.83 & $4 \cdot 25$ & $4 \cdot 19$ & 4.46 \\
\hline 17 & 1.74 & $2 \cdot 30$ & $2 \cdot 74$ & 3.04 & $3 \cdot 36$ & 3.85 & 4.03 & $4 \cdot 30$ & $4 \cdot 24$ \\
\hline 18 & 1.74 & $2 \cdot 55$ & 3.11 & 3.60 & $4 \cdot 15$ & 4.63 & 4.91 & $5 \cdot 08$ & $5 \cdot 38$ \\
\hline 19 & 1.80 & $2 \cdot 29$ & 2.95 & 3.47 & 3.93 & 4.39 & 4.55 & 5.00 & $5 \cdot 69$ \\
\hline 20 & $1 \cdot 80$ & 2.39 & 2.75 & $3 \cdot 19$ & $3-70$ & 4.04 & 4.44 & 4.71 & 4.92 \\
\hline 21 & 1.88 & $2 \cdot 40$ & $3 \cdot 16$ & 3.55 & 3.96 & $4 \cdot 74$ & 4.90 & 5.62 & $6 \cdot 30$ \\
\hline 22 & 1.81 & 2.49 & $2 \cdot 84$ & $3 \cdot 23$ & $3-74$ & $4 \cdot 49$ & $4 \cdot 77$ & $5 \cdot 17$ & $5 \cdot 53$ \\
\hline 23 & 1.68 & $2 \cdot 17$ & 2.72 & 3.40 & 3.50 & 3.63 & $4 \cdot 37$ & 4.79 & 4.85 \\
\hline 24 & 1.77 & $2 \cdot 28$ & 2.79 & 3.05 & $3 \cdot 47$ & 3.94 & $4 \cdot 57$ & $4 \cdot 59$ & 5.03 \\
\hline 25 & 1.83 & $2 \cdot 39$ & 2.94 & $3 \cdot 39$ & $4-23$ & 4.45 & $4 \cdot 40$ & 4.96 & 5.69 \\
\hline 26 & 1.86 & 2.55 & 3.09 & 3.65 & 4.01 & $4 \cdot 31$ & 5.02 & $5 \cdot 40$ & $5 \cdot 29$ \\
\hline 27 & 1.88 & 2.44 & 3.07 & $3 \cdot 33$ & 3-79 & $4 \cdot 01$ & $4 \cdot 70$ & $5 \cdot 26$ & 5.49 \\
\hline 28 & 1.76 & 2.45 & 2.97 & 3.48 & 3.97 & $3 \cdot 86$ & 4.42 & $4 \cdot 64$ & 4.92 \\
\hline 29 & 1.65 & $2 \cdot 50$ & $3 \cdot 12$ & 3.43 & 405 & 4.56 & $4 \cdot 43$ & 5.00 & 5.57 \\
\hline 30 & 1.83 & 2.44 & 2.98 & 3.52 & $4-25$ & $4 \cdot 41$ & $4 \cdot 62$ & 5.08 & $5 \cdot 51$ \\
\hline 31 & 1.75 & 2.57 & 2.90 & 3.39 & 3.75 & $4 \cdot 44$ & $4 \cdot 61$ & 5.02 & $5 \cdot 24$ \\
\hline 32 & 1.69 & 2.35 & 2.82 & 3.38 & 3.92 & $4 \cdot 20$ & $4 \cdot 65$ & 5.05 & $5 \cdot 21$ \\
\hline 33 & 1.74 & $2 \cdot 37$ & $3 \cdot 10$ & 3.82 & 4.09 & $4 \cdot 13$ & $4 \cdot 62$ & $5 \cdot 12$ & $5 \cdot 34$ \\
\hline 34 & 1.82 & 2.44 & $2 \cdot 88$ & 3.45 & 4.03 & $4 \cdot 14$ & $4 \cdot 26$ & $4 \cdot 81$ & $5 \cdot 29$ \\
\hline
\end{tabular}


consistent with the known picture of monsoon spatial variability. Finally, these results for subdivisions are similar to our earlier result for the all-India rainfall (Satyan 1988). This similarity in the dynamics of the large and small spatial scale systems is in agreement with our earlier findings that summer monsoon variability on the inter-annual and longer time scales is essentially due to fluctuations in the large-scale stationary flow patterns over the monsoon region (Keshavamurty et al 1978; Satyan et al 1980). Next we see that the critical value of the embedding phase space dimension $n$ beyond which $d$ saturates is around 10. From our discussion in $\$ 2$ this number provides an upper bound to the number of independent variables needed to model the dynamics. The minimum number of independent variables required to model the dynamics is given by the integer next to the attractor dimensionality for the subdivision.

\section{Acknowledgements}

The author is thankful tu Dr B Parthasarathy for providing the subdivisional seasonal rainfall data for the poriod 1871-1986 and to Prof. R N Keshavamurty for many useful discussions. Thanks are also due to Mr V Krishnakumar for assistance.

\section{References}

Fraedrich K 1986 Estimating the dimensions of weather and climate attractors; J Atmos. Sci. 43 419-432 Grassberger P and Procaccia I 1983 Characterization of strange attractors; Phys. Rev. Lett. 50 346-349

Hense A 1987 On the possible existence of a strange attractor for the southern oscillation; Beitr. Phys. Atmos. $6034-47$

Keshavamurty R N, Satyan V and Goswami B N 1978 Indian summer monsoon cyclogenesis and its variability; Nature (London) 274 576-578

Lorenz E N 1963 Deterministic nonperiodic flow; J Atmos. Sci. 20 130-141

Lorenz E N 1984 Irregularity - a fundamental property of the atmosphere; Tellus A36 98-110

Mooley D A and Parthasarathy B 1984 Fluctuations in all-India summer monsoon rainfall during 1871-1978; Climatic Change 6 287-301

Nicolis C and Nicolis G 1984 Is there a climatic attractor? Nature (London) 311 529-532

Satyan V 1988 Is there an attractor for the Indian summer monsoon? Proc. Indian Acad. Sci. (Earth Planet. Sci.) 97 49-52

Satyan V, Keshavamurty R N, Goswami B N, Dash S K and Sinha H S S 1980 Monsoon cyclogenesis and large scale flow patterns over south Asia; Proc. Indian Acad. Sci. (Earth Planet. Sci.) 89 277-292 